\title{
ABNORMAL SPERMATOZOAN CHROMATIN IN INFERTILE BULLS
}

\author{
P. J. McGOSKER* \\ Department of Animal Husbandry, University of Queensland, St. Lucia, Queensland, \\ Australia
}

(Received 16th September 1968)

Morphological abnormalities of spermatozoa have long been a subject of study, and a small percentage of abnormal spermatozoa is to be seen in most ejaculates. Such abnormalities were first described using light microscopy and later using the electron microscope. In some later studies, specific morphological changes have been clearly demonstrated, e.g. acrosome abnormalities in boar spermatozoa (Bane, 1961; Bane \& Nicander, 1966), 'knobbed sperm' defect (Blom \& Birch-Anderson, 1962) and 'pouch formations' in the nucleus (Bane \& Nicander, 1965). Some of these changes have been associated with sterility.

This preliminary communication describes changes in the nuclear chromatin of the spermatozoa of infertile bulls which may be of interest in relation to current work on the maturation of nuclear chromatin. The major abnormalities observed were a granular appearance of the nuclear chromatin in a high percentage of spermatozoa, and intranuclear spaces.

Semen was collected by electroejaculation from two $F_{2}$ generation Bos taurus $\times$ Bos indicus bulls. Spermatozoa were concentrated by centrifugation and fixed in $4.16 \%$ glutaraldehyde in sodium cacodilate at $\mathrm{pH} 7.2$ (Sabatini, Bensch \& Barrnett, 1963) for 2 to 3 hours. Pellets of spermatozoa were washed in two changes of buffered sucrose, post-fixed in $1 \%$ osmium tetroxide for $1 \frac{1}{2} \mathrm{hr}$, dehydrated in ascending concentrations of ethyl alcohol and embedded in Araldite (Glauert, Rogers \& Glauert, 1956). Sections were cut with glass knives using an L.K.B. 'Ultratone', supported on copper grids and stained with $5 \%$ uranyl acetate and Reynolds lead citrate (Reynolds, 1963). Material was viewed on either a Siemens Elimskop I or IA Microscope.

Granulation of the chromatin was the most striking and probably the most important morphological abnormality in the spermatozoa of these bulls. Although no estimate was made of the percentage of abnormal heads of this type, it is evident from Pl. 1, Fig. 1 that the majority showed granulation of the chromatin varying from very fine to coarse. The remainder of the nuclei showed apparently normal condensation of nuclear chromatin, being dense and homogeneous, but both granular and non-granular forms showed spaces in the chromatin.

Granular nuclear chromatin does not appear to have been described previously in ejaculated spermatozoa, but it is well known that the chromatin of

* Present address: Department of Veterinary Clinical Studies, University of Queensland. 
spermatids is granular in appearance when studied under comparable conditions with the electron microscope (Fawcett, 1958). The process of condensation of the nuclear chromatin of the spermatozoa of various species is currently being studied and the spermatozoa described here are probably spermatids which have undergone a defect in the condensation of the chromatin. The microspectrophotometric studies of Gledhill, Gledhill, Rigler \& Ringërtz (1966) suggest that the condensation of the chromatin involves the firm binding of the nuclear protein to DNA. They suggest that this causes a contraction of the DNA helix and therefore a shrinking of the structure of the nucleus.

Various types of spaces within the nucleus of both normal and abnormal spermatozoa have been described in the literature. Thus, small vacuoles appear to be present in the nucleus of normal spermatozoa of various species including cattle (Fawcett, 1958), but other types of spaces within the nucleus are definitely considered abnormal. The invaginations of the nuclear envelope of bovine and porcine spermatozoa described by Bane \& Nicander (1965) are in the latter category.

The spaces seen in these spermatozoa are of two types. The first of these types (Pl. 1, Fig. 2) appears to be comparable to the 'pouch' formation described by Bane \& Nicander (1965) in that it contains structures, appears to have a limiting membrane and in some instances is an invagination. Bane \& Nicander associate these spaces with acute degenerative processes. The second type of space possesses neither contents nor limiting membrane and is apparent both in heads with normal chromatin and in those with diffusely granular chromatin (Pl. 1, Fig. 2). These spaces could be artifacts caused by centrifugation; however, specimens of semen from normal bulls, rams, dogs and cockerels concentrated in the same way in the author's laboratory do not show comparable changes, so it is unlikely that centrifugation caused these spaces, at least in those nuclei with normal chromatin. On the other hand, defective condensation of the chromatin may have predisposed to cavity formation on centrifugation in those nuclei with granular chromatin; but in those nuclei with apparently normal chromatin in which spaces were observed, there may have been modifications in the structure of the chromatin which were not revealed by this technique.

It is tentatively concluded, therefore, that the sterility in these bulls was caused by the grossly abnormal nuclear structure of the spermatozoa and that these abnormalities may be a result of a defect in the maturation of spermatids. It is not possible to say whether this is a temporary or permanent condition, but this study is being continued.

\section{EXPLANATION OF PLATE 1}

Frg. 1. The frequency of the abnormalities described is illustrated. Approximately $70 \%$ of the sperm heads have abnormally granular chromatin. Spaces of different types are also apparent in the chromatin of various nuclei.

FIG. 2. The two different types of space within nuclei with relatively normal chromatin ( 1 and 2 ) and nuclei with granular chromatin ( 3 and 4 ) are illustrated. Spermatozoon 1 contains a space without contents or membrane, while 2 contains an invagination with contents. Spermatozoa 3 and 4 both contain invaginations and spaces; in these, the invaginations (A) are sectioned in such a way that they appear as spaces. 
PLATE 1

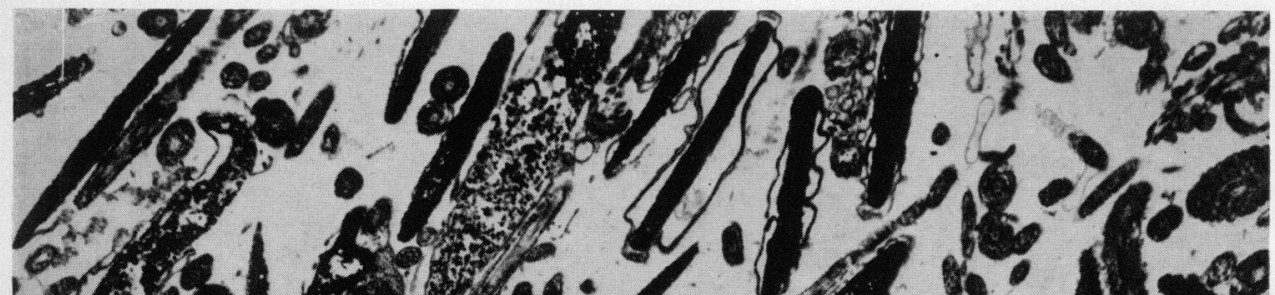

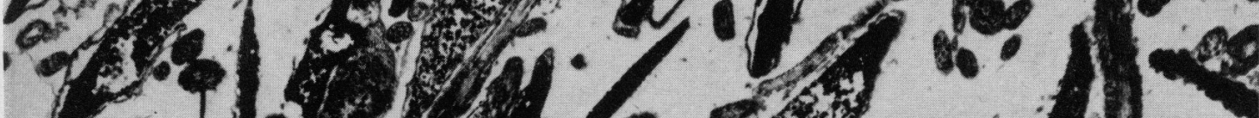
i.

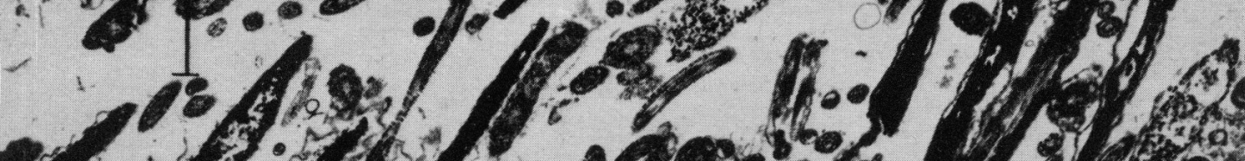

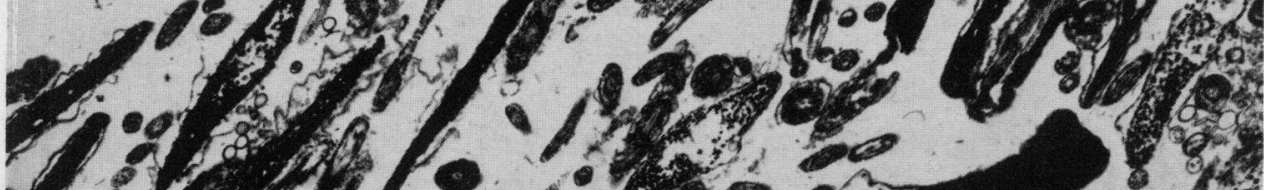
(2)

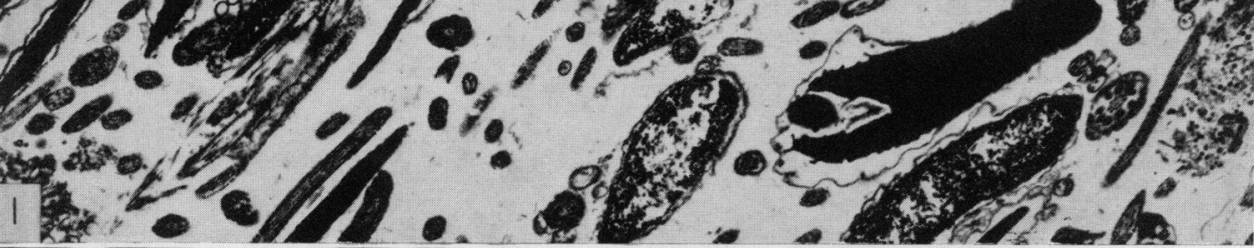

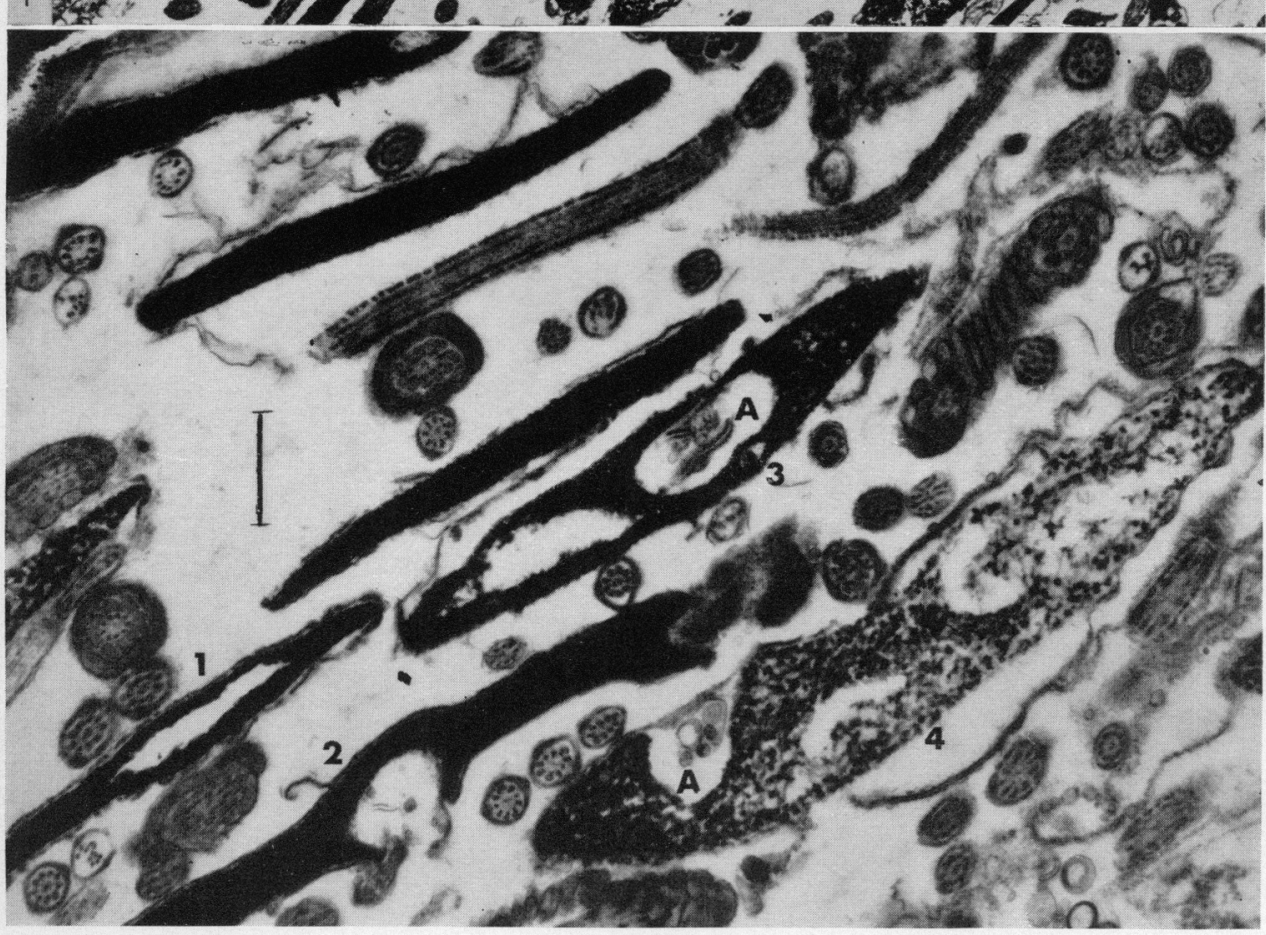


The assistance of Professor D. F. Dowling, the technical assistance of D. Gowanlock and the comments of Professor J. L. Hancock and Professor A. Bane are gratefully acknowledged.

\section{REFERENCES}

BANE, A. (1961) Acrosomal abnormality associated with sterility in the boar. Proc. IVth int. Congr. Anim. Reprod., The Hague, p. 810.

BANE, A. \& NiCANDER, L. (1965) Pouch formation by invagination of the nuclear envelope of bovine and porcine sperm as a sign of disturbed spermiogenesis. Nord. Vet. Med. 17, 628.

BANE, A. \& NicANDER, L. (1966) Electron and light microscopical studies of spermateliosis in a boar with acrosome abnormalities. F. Reprod. Fert. 11, 133.

Blom, E. \& Birch-Anderson, A. (1962) Ultrastructure of the sterilizing "knobbed sperm" defect in the bull. Nature, Lond. 194, 989.

FAwCETT, R. W. (1958) The structure of mammalian spermatozoon. Int. Rev. Cytol. 7, 195.

Glauert, A. M., Rogers, G. E. \& Glauert, R. H. (1956) A new embedding medium for electronmicroscopy. Nature, Lond. 178, 803.

Gledhill, B. L., Gledhill, M. P., Rigler, R. \& Ringërtz, N. R. (1966) Changes in deoxyribonucleoprotein during spermiogenesis in the bull. Expl Cell Res. 41, 652.

REynolds, E. S. (1963) The use of lead citrate at high $\mathrm{pH}$ as an electron-opaque stain in electron microscopy. F. Cell Biol. 17, 208.

Sabatini, D. D., Bensch, K. \& Barrnett, R. J. (1963) Cyto-chemistry and electron microscopy. The preservation of cellular ultrastructure and enzymatic activity by aldehyde fixation. 7 . Cell Biol. 17, 19. 